\title{
A Method to Extract P300 EEG Signal Feature Using Independent Component Analysis (ICA) for Lie Detection
}

\author{
P. A. Antasaria, W. Caesarendra ${ }^{b}$, A. Turnipc ${ }^{c}$, and I.S. Aisyah ${ }^{d}$ \\ a,b Mechanical Engineering, Diponegoro University, Semarang, Indonesia \\ e-mail :w.caesarendra@gmail.com \\ 'Instrumentation Laboratory, Indonesian Institute of Science, Bandung, Indonesia \\ ${ }^{d}$ Mechanical Engineering, University of Muhammadiyah Malang, Malang, Indonesia
}

\section{Abstract}

The progress of today's technology is growing very quickly. This becomes the motivation for the community to be able to continue and provide innovations. One technology to be developed is the application of brain signals or called with electroencephalograph (EEG). EEG is a non-invasive measurement method that represents electrical signals from brain activity obtained by placement of multiple electrodes on the scalp in the area of the brain, thus obtaining information on electrical brain signals to be processed and analyzed. Lie is an act of covering up something so that only the person who is lying knows the truth of the statement. The hidden information from lying subjects will elicit an EEG-P300 signal response using Independent Component Analysis (ICA) in different shapes of amplitude that tends to be larger around $300 \mathrm{~ms}$ after stimulation. The method used in the experiment is to invite subject in a card game so that the process can be done naturally and the subject can well stimulated. After the trials there are several results almost all subjects have the same frequency on the frequency of 24-27 Hz. This is a classification of beta waves that have a frequency of $13-30 \mathrm{~Hz}$ where the beta wave is closely related to active thinking and attention, focusing on the outside world or solving concrete problems.

Keywords: EEG signal; Lie detection; ICA; P300 feature

\section{INTRODUCTION}

EEG is a non-invasive measurement method that represents electrical signals from brain activity obtained by placement of multiple electrodes on the scalp in the area of the brain, thus obtaining information on electrical brain signals to be processed and analysed [1]. In the medical field EEG can be used to investigate epilepsy, Alzheimer's, schizophrenia and continue to be improved in more effective EEG applications.

The EEG application for lie detection has been studied since the 1990s as an alternative to lie detection methods using polygraph [2]. In polygraph-based lie detection, testing is done by observing the physiological responses of subjects such as respiration and blood pressure [3]. This method has a weakness that is easily deceived results through the process of anticipation (countermeasure) of the person being tested; For example, subjects may consume drugs to stabilize blood pressure. Based on these problems, the EEG is examined as one of the alternative methods for lie detection, with the premise that EEG signals will be more difficult to fool than physiological responses to polygraphy.

Event-related potential (ERP) or evoked potential is an electrical signal that appears in the brain in response to certain stimuli. ERP tends to have low amplitude $(<5 \mu \mathrm{V})$ compared with the EEG background signal (50-600 $\mu \mathrm{V})$ [4], so its appearance cannot be 
identified through normal EEG recordings. P300 is an ERP with a positive deflection of EEG that appears approximately $300 \mathrm{~ms}$ after stimulus.P300 is a description of the cortical electrophysiological activity that regulates the activity of care, differentiation of problems, decision-making, memory, and integration. P300 not only appears on the vision alone. ERP auditory P300 shows an object measured from the central auditory function.

Band Pass Filter is a filter that only passes signals whose frequencies are listed in the band or certain band pass. Frequency of signals that are below the frequency band or above, cannot be passed or muted by the Band Pass Filter circuit. ICA is a method used in separating EEG signals from artefact estimation of a signal without the need for a reference channel. In MATLAB this ICA is used to extract the feature of EEG signal data. In this study, the right methodology using the card as a medium to find out the input of brain signals from subject where the subject will think honestly or lie.

\section{Methodology}

This chapter contains important research data, equipment used and research location. The research method used is explained briefly (can be made in the form of flow chart). The new method or modified method is described in detail.

\subsection{EEG signal processing device}

In this study there are some tools used in experimental data retrieval as well as data processing done. For the EEG signal acquisition phase in this study, the hardware used is the Mitsar-202 EEG Amplifier. To record an EEG signal using this amplifier, the amplifier device is connected to a PC using USB. Furthermore, the amplifier is operated via WinEEG software on the PC. Furthermore, there is an Electro-Cap is a hat-shaped device that serves to facilitate the placement of EEG electrodes. This device consists of a number of tin electrodes connected to the amplifier via adapter [5]. Electro-Cap display can be observed in Figure 2.1.

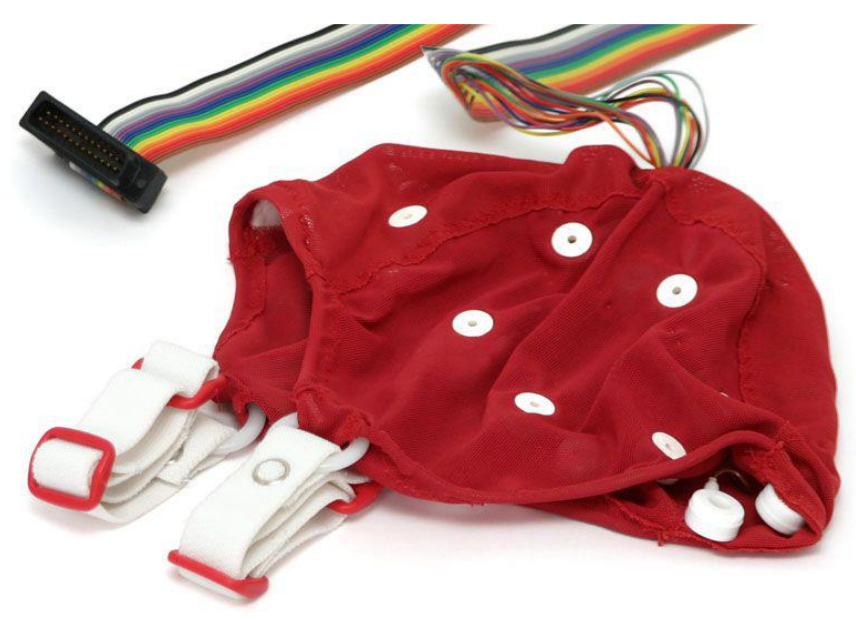

Figure 2.1 Electro-cap unit

\subsection{Experimental method}

The flow process of experimental and signal processing method used for detecting lies using the EEG-P300 feature is presented in Figure 2.2.

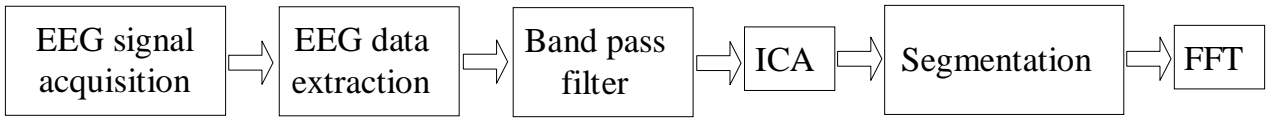

Figure 2.2 The experimental and signal processing method for lie detection 
In this experiment, the stimuli used are five visual questions asked to each subject to observe the emergence of ERP on the naracoba EEG signal. These four subjects are divided into two groups where; The first "innocent" or "honest" group holding the number cards and the second group "guilty" or "lying" with a picture card as presented in Table 2.1. These four subjects will be followed by a card game whose procedure is known only to the researcher for the more leverage expected results.

Table 2.1 Subjects list EEG-based lie detection experiment

\begin{tabular}{cccc}
\hline Subject & Age (years) & Gender & Group \\
\hline 1 & 26 & Male & Honest \\
2 & 24 & Male & Honest \\
3 & 22 & Male & Lie \\
4 & 24 & Male & Lie \\
\hline
\end{tabular}

Prior to the experiment, each subject were asked to spend money of $R p 20,000$. The researcher held the total amount of Rp 80,000. Researcher have four cards in which two of them are picture cards. The researcher instructed that researcher would distribute each card to the subject. For subjects who gets a picture card will be the perpetrator who earned the money that has been collected previously, but enforced a condition where the subject should not be known by other subjects that he has a picture card. When given a question stimulus, the subject must retain the card. If a subject who gets a picture card cannot keep the card then the money that has been collected will be the property of a subject who has a number card. Only the researcher knows the distribution of the card so that the researcher knows who gets the picture card and the number card. The researcher distributes the card to each participant and the participants can see the card unnoticed by the other participants. Next, one by one the participants paired EEG and asked five questions by other subjects. Each questioner who asks and answers defends his argument that the money they have collected belongs to another subjects. At the time of being asked the questions the participants only answer "yes" or "no" and write the answer on the board. As long as the question asked by the subject, the researchers record the time when giving subjects questions and answers, as well as the contents of the given questions.

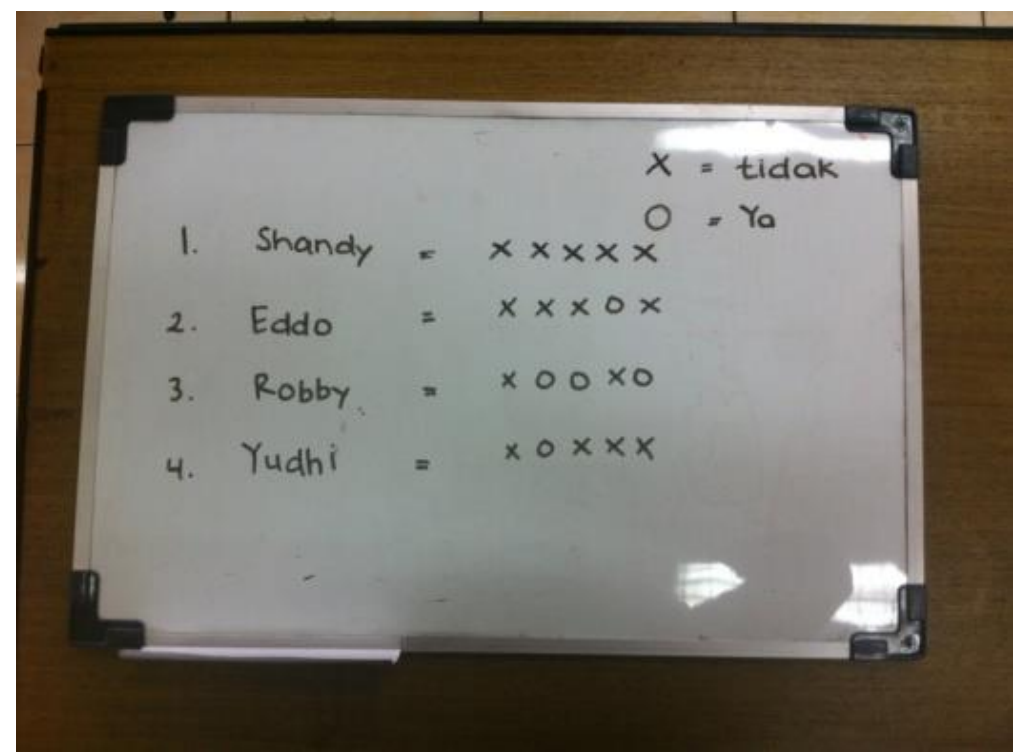

Figure 2.3 The answer results of each subject. 


\subsection{EEG signal recording}

EEG signal recording is performed on three electrodes that have the following areas: frontal $(\mathrm{Fz})$, central $(\mathrm{Cz})$, parietal $(\mathrm{Pz})$. These three channels are the channels used in previous P300 component-based lie detection research [6]. During the recording experiment, allocated time for each subject is one minute for five questions. The signal recording process can be observed in Figure 2.4.

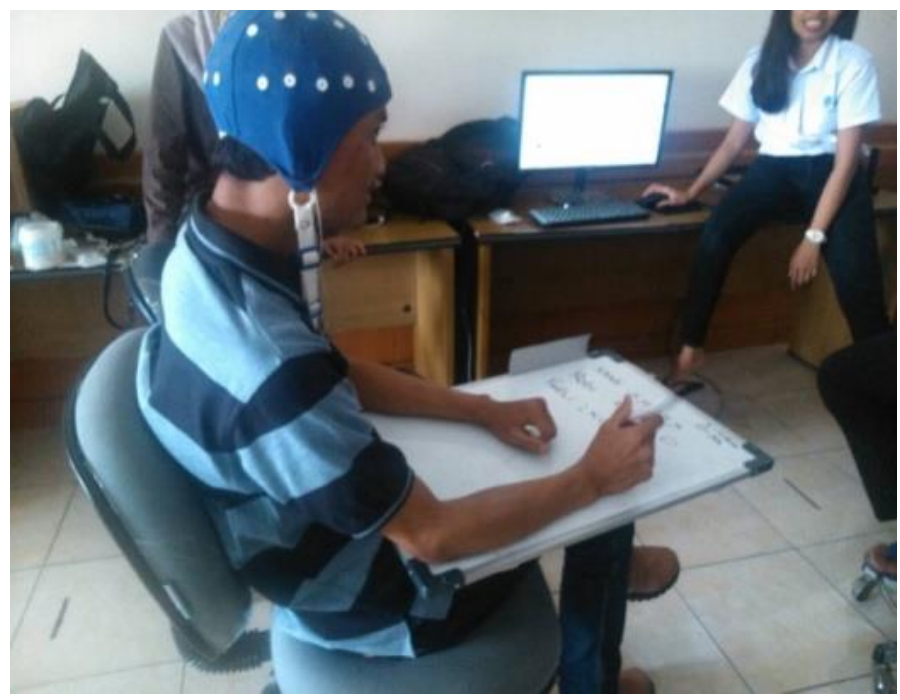

Figure 2.4 The process of recording the signal on subject \#4.

\subsection{Data processing}

Data extraction is done with the purpose of changing the file *.EDF into file format ${ }^{*}$. $m$ to be processed on MATLAB software. Extraction can be done using the EEGLAB toolbox. At the time of recording EEG signals there are many artifacts mixed on the EEG signal caused by the movement on subject, blink of the eye, and so forth. The number of artifacts makes signal processing difficult to do accurately. Therefore, pre-processing needs to be done so that the signal can be cleaned and processed further. In preprocessing this time using band-pass filter corrections.

In this study, the EEG rough signal with a sampling frequency of $500 \mathrm{~Hz}$ will be filtered with a frequency range of $0.3-30 \mathrm{~Hz}$; The frequency range is commonly used for ERP analysis [6]. The filtering process is done by entering the data encoding for the band pass filter in the MATLAB command line. In the recorded EEG signal, data on a channel is performed separating signals from artifacts. ICA serves to parse the data mix by recovering the sources of the recorded signal. Fast Fourier Transform is done in order to transform signals in time domain into signals in the frequency domain. Each subject has three channels in which each channel has an average amplitude of five answers as well as the average frequency at the maximum amplitude. This is done so that the P300 applied to this experiment can be seen clearly.

\section{Results AND Discussion}

\subsection{Analysis of EEG signal}

Stimulus question given to subject, EEG signal recorded and result of measurement in the form of raw data from each condition on three channel for subject \# 4 can be seen in Figure 3.1. 


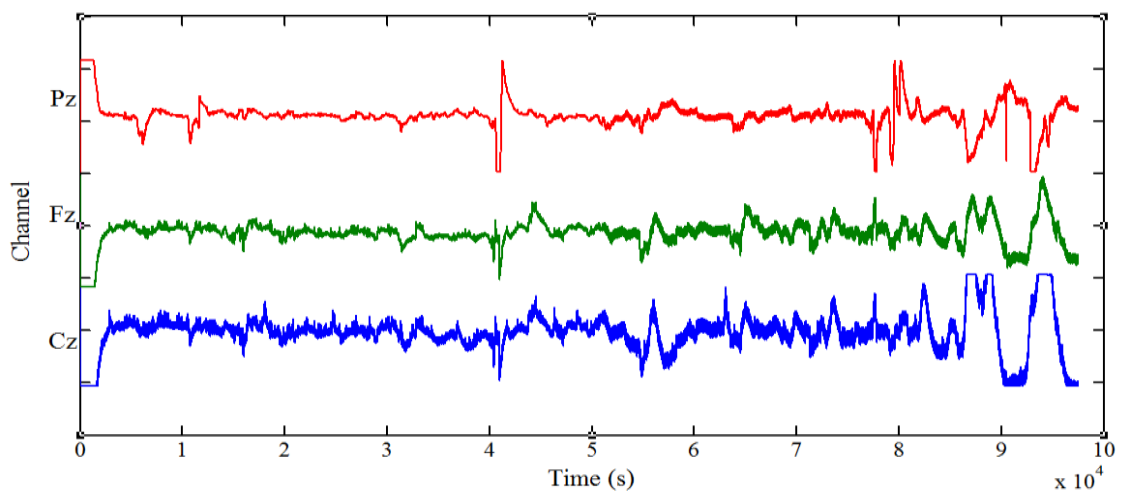

Figure 3.1 EEG signal subject \#4.

\subsection{The processed EEG signal result using BPF method}

The next step after the recording of the raw data signal is to pre-process by applying the band-pass filter. In the signal band-pass filter results, some segments are not good recording. One cause is because subject make movement and think so subject not focus on given question.

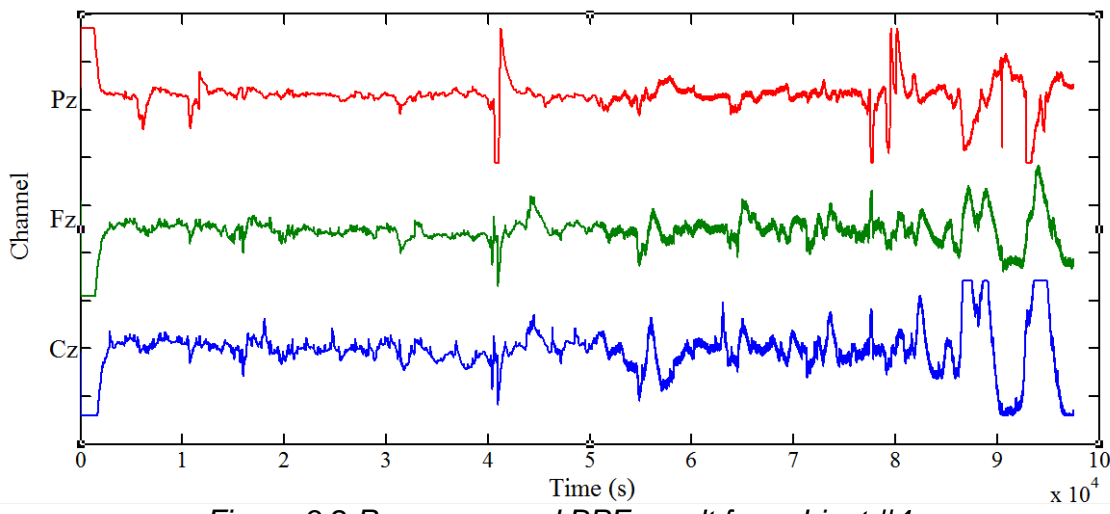

Figure 3.2 Pre-processed BPF result for subject \#4.

\subsection{The processed EEG signal result using ICA method}

After the ICA process, it can be observed that the artefacts on this signal are not completely lost. This is due to the movement of subject that makes the artefacts contained in the signal cannot be filtered thoroughly.

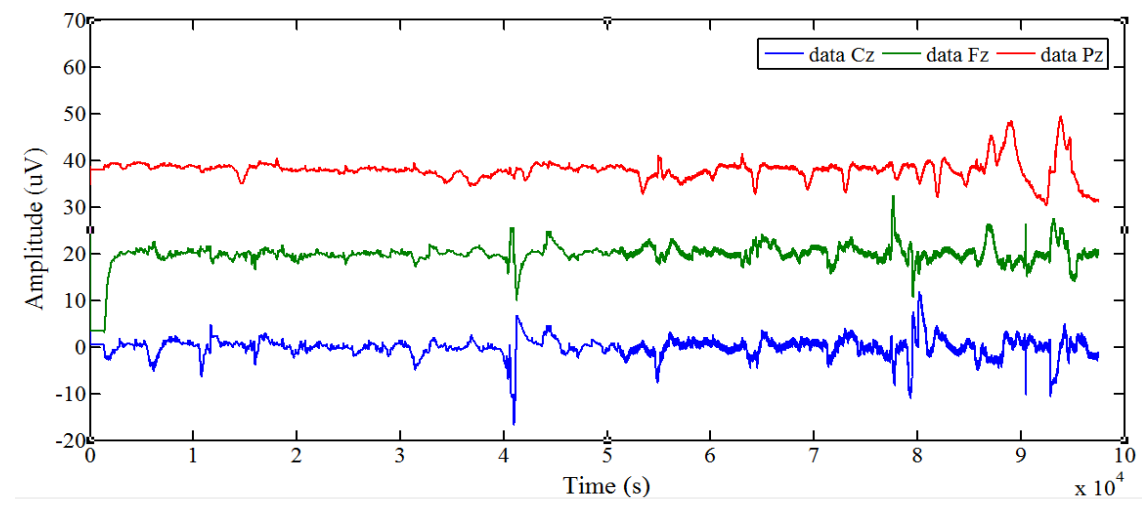

Figure 3.3 ICA result for subject \#4. 


\subsection{Data segmentation result}

In this process, it is clear that the target and non-targeted subject \#2 has almost the same signal waves that the P300 is difficult to see. This shows the subject saying honestly. While target and non-target signal waves on subject \#4 show a significant difference where the subject responds. So at the target found P300 after 331 ms with amplitude $0.719 \mu \mathrm{V}$ as shown in the Figures 3.4 and 3.5.

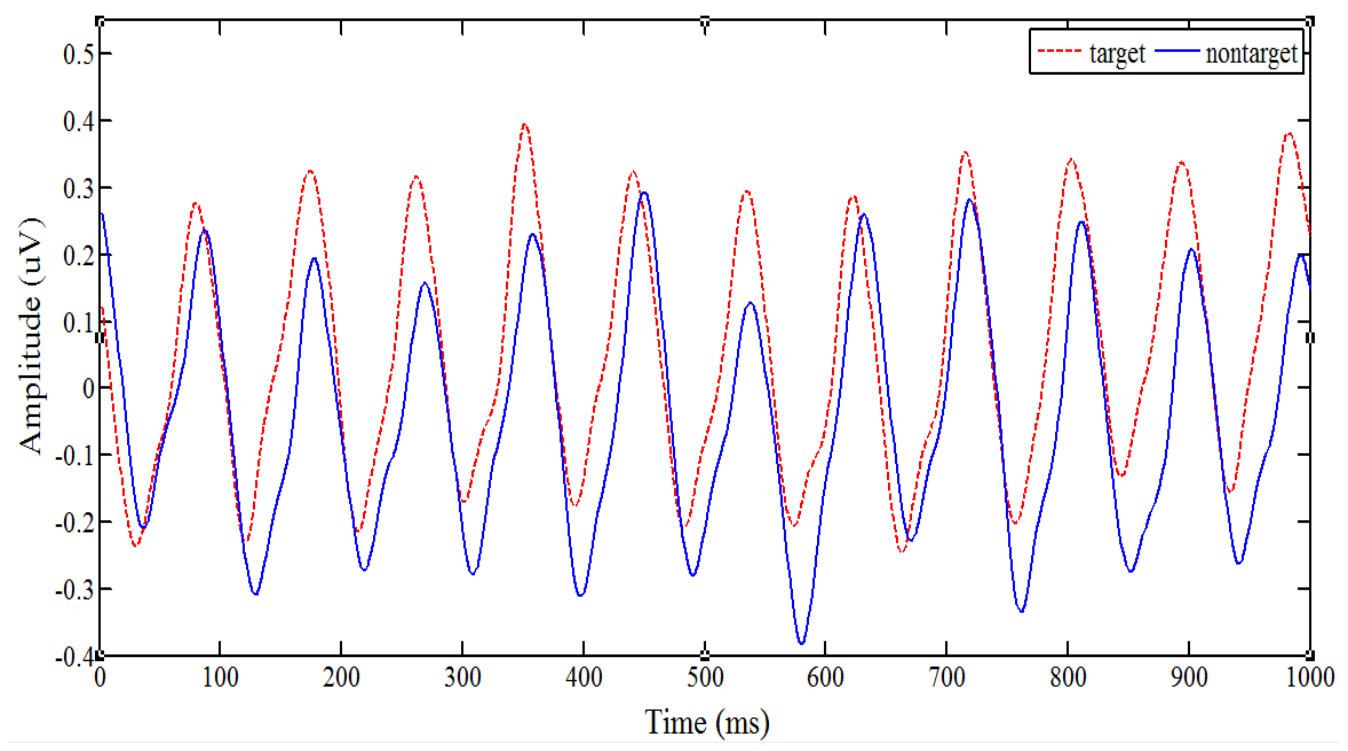

Figure 3.4 Non-target Pz channel target result on subject \#2 (honest).

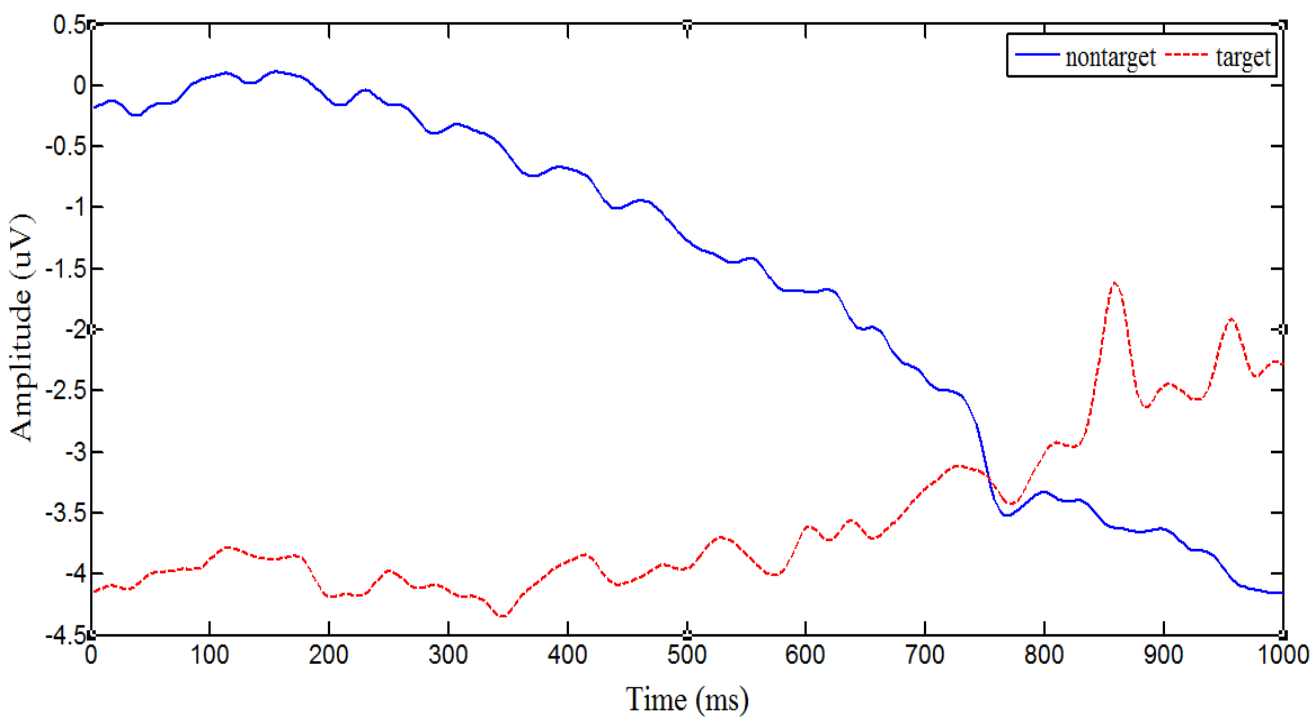

Figure 3.5 Non-target Pz channel target result on subject \#4 (lie).

Here is the average table of amplitude, latency, and average frequency of the four subjects. Almost all subjects have the same average frequency on the Beta frequency wave. However, there is a frequency difference shown as the example of subject \# 3 which is an error factor that occurred during the experiment, in which the subject condition was less concentrated during the experiment. 


\begin{tabular}{|c|c|c|c|c|c|c|c|c|c|}
\hline \multirow{2}{*}{ Subject } & \multicolumn{3}{|c|}{$C z$} & \multicolumn{3}{|c|}{ Fz } & \multicolumn{3}{|c|}{ Pz } \\
\hline & $A(u V)$ & (ms) & $\mathbf{F ( H z )}$ & $A(u V)$ & L(ms) & $\overline{F(H z)}$ & $A(u V)$ & L(ms) & $\mathbf{F}(\mathrm{Hz})$ \\
\hline 1 & 3.80 & 79 & 24 & 0.68 & 319 & 24 & 1.53 & 128 & 27 \\
\hline 2 & 0.52 & 35 & 24 & 0.66 & 337 & 24 & 0.46 & 109 & 24 \\
\hline 3 & 1.90 & 333 & 7 & 0.82 & 85 & 5 & 1.67 & 330 & 5 \\
\hline 4 & 0.39 & 392 & 27 & 0.39 & 99 & 27 & 0.71 & 331 & 27 \\
\hline
\end{tabular}

Table 3.2(a): Cz.

\begin{tabular}{cccc}
\hline Subject & \multicolumn{4}{c}{$\mathbf{A}(\mathbf{u V}) \mathbf{L}(\mathbf{m s})$} & $\mathbf{F}(\mathbf{H z})$ \\
\hline 1 & 3.80 & 79 & 24 \\
2 & 0.52 & 35 & 24 \\
3 & 1.90 & 333 & 7 \\
4 & 0.39 & 392 & 27 \\
\hline \multicolumn{4}{c}{ Table $3.2(b): F z$} \\
\hline Subject & $\mathbf{A}(\mathbf{u V})$ & $\mathbf{L}(\mathbf{m s})$ & $\mathbf{F}(\mathbf{H z})$ \\
\hline 1 & 0.68 & 319 & 24 \\
2 & 0.66 & 337 & 24 \\
3 & 0.82 & 85 & 5 \\
4 & 0.39 & 99 & 27 \\
\hline \multicolumn{5}{c}{ Table $3.2(c): P z$} & \\
\hline Subject & $\mathbf{A}(\mathbf{u V})$ & $\mathbf{L}(\mathbf{m s})$ & $\mathbf{F}(\mathbf{H z})$ \\
\hline 1 & 1.53 & 128 & 27 \\
2 & 0.46 & 109 & 24 \\
3 & 1.67 & 330 & 5 \\
4 & 0.71 & 331 & 27 \\
\hline
\end{tabular}

\section{CONCLUSION}

From the experiment result, two conclusion can be drawn:

1. The amplitude of the "honest" narrator has uniform amplitude on all three channels so that the P300 cannot be observed. The amplitude of the narrator "lying" has a spike after passing $300 \mathrm{~ms}$ according to the method used is P300.

2. Almost all subjects have the same average frequency that is Noor \# 1 at $24 \mathrm{~Hz}$, Naracoba \# 2 at $24 \mathrm{~Hz}$, and subject \#4 at $27 \mathrm{~Hz}$. This is a classification of beta waves that have a frequency of $13-30 \mathrm{~Hz}$ where this wave is closely related to active thinking and attention, focusing on the outside world or solving concrete problems. However subject \# 3 has an average frequency difference of $5 \mathrm{~Hz}$ on channel Fz and $\mathrm{Pz}$ and $7 \mathrm{~Hz}$ on the $\mathrm{Cz}$ channel which is a classification of theta waves which has a frequency range of 4-8 Hz. These waves emerge from emotional stress and also because of the unconscious, inspirational, and meditative. This error can occur due to several factors one of which is the lack of concentration of the subject.

\section{REFERENCES}

[1] S. Sanei and J. A. Chambers, EEG Signal Processing, vol. 1. 2007.

[2] L. A. Farwell and E. Donchin, "The Truth Will Out: Interrogative Polygraphy ('Lie Detection') With Event- Related Brain Potentials," Psychophysiology, vol. 28, no. 5, pp. 531-547, 1991. 
[3] L. Saxe, D. Dougherty, and T. Cross, "The validity of polygraph testing: Scientific analysis and public controversy," Am. Psychol., vol. 40, no. 3, pp. 355-366, 1985.

[4] D. L. Hawksworth, "John Webster: publications 1951-2004," Mycol. Res., vol. 109, no. 5, pp. 649-654, 2005.

[5] Anonymous, "Electro-cap International," 12 February, 2016.

[6] V. Abootalebi, M. H. Moradi, and M. A. Khalilzadeh, "A new approach for EEG feature extraction in P300-based lie detection," Comput. Methods Programs Biomed., vol. 94 , no. 1 , pp. 48-57, 2009. 